\title{
Quelques réflexions sur la recherche méthodologique liée aux opérations de développement
}

\section{Pierre Lefèvre}

\author{
(2) OpenEdition \\ Journals \\ Édition électronique \\ URL : http://journals.openedition.org/apad/2493 \\ DOI : 10.4000/apad.2493 \\ ISSN : 1950-6929 \\ Éditeur \\ LIT Verlag
}

\section{Édition imprimée}

Date de publication : 1 décembre 1993

\section{Référence électronique}

Pierre Lefèvre, «Quelques réflexions sur la recherche méthodologique liée aux opérations de développement », Bulletin de l'APAD [En ligne], 6 | 1993, mis en ligne le 10 mars 2008, consulté le 07 septembre 2020. URL : http://journals.openedition.org/apad/2493 ; DOI : https://doi.org/10.4000/ apad.2493

Ce document a été généré automatiquement le 7 septembre 2020

Bulletin de I'APAD 


\title{
Quelques réflexions sur la recherche méthodologique liée aux opérations de développement
}

\author{
Pierre Lefèvre
}

Introduction

1 La recherche méthodologique relative aux opérations de développement a été identifiée comme un des domaines à privilégier par une anthropologie du changement social et du développement. Une contribution accrue de l'anthropo-sociologie à ce type de recherche est considérée comme l'un des moyens de combler le fossé qui s'est créé entre les théoriciens du développement et l'anthropo-sociologie académique d'une part et, d'autre part, les travailleurs des sciences humaines engagés sur le terrain dans des interventions concrètes. La recherche méthodologique est également perçue comme pouvant augmenter la crédibilité de l'anthropo-sociologie auprès des opérateurs de développement et des techniciens sectoriaux qui critiquent, à tort ou à raison, son caractère peu opérationnel.

2 Je propose ici quelques réflexions sur ce type de recherche élaborées à la lumière de l'expérience accumulée comme sociologue au sein de l'équipe interdisciplinaire que constitue l'Unité de Nutrition de l'Institut de Médecine Tropicale d'Anvers (IMT). La recherche méthodologique est, en effet, depuis plusieurs années, un des domaines d'action privilégiés de l'Unité. En particulier, l'Unité a pu travailler au développement d'une méthodologie d'évaluation globale et participative de projets de développement complexes, à composante nutritionnelle ${ }^{1}$, dans le cadre du programme Science et Technologie pour le Développement (STD) de la Communauté Européenne. Mon objectif est de mettre en évidence certaines caractéristiques - mais également certaines contraintes - de ce type de recherche pour ceux qui seraient tentés par l'aventure. Je n'envisage pas ici la situation du chercheur isolé, qui serait amené à développer ou à adapter des outils à l'occasion d'une activité de consultation ponctuelle.

Une demande réelle mais confuse... 
3 Dans le domaine nutritionnel et plus généralement dans le secteur de la santé publique, il existe une demande effective et importante en matière de "méthodes". Cette demande émane des organisations internationales, des organismes de coopération bilatéraux ou multilatéraux et de certaines ONG. Cette demande s'adresse généralement à des centres de recherche privés ou publics et aux universités ou instituts du secteur. Au sein du secteur santé, il est également de plus en plus admis que l'anthropo-sociologie a un apport spécifique à apporter dans ce domaine. Un récent colloque tenu au Centre International de l'Enfance à Paris ${ }^{2}$, consacré à la recherche sur les systèmes de santé, et qui réunissait l'ensemble des équipes de recherche financées par le programme STD, a illustré clairement non seulement une reconnaissance du rôle de l'anthropo-sociologie mais également la présence effective de la discipline dans de nombreux projets de recherche en cours.

4 Une des caractéristiques de la demande adressée à l'anthropo-sociologie, qu'elle émane d'organisations ou de techniciens engagés dans le développement, est qu'elle s'exprime souvent de manière à la fois confuse et excessive. En pratique, les techniciens sectoriaux et les opérateurs de développement rencontrent de nombreux problèmes dans la mise en œuvre de leurs interventions. Les problèmes non techniques sont étiquetés comme relevant de la compétence des sciences humaines mais sont rarement définis clairement. A l'image de leur propre pratique, les techniciens attendent du sociologue des solutions techniques simples et efficaces aux problèmes rencontrés. La demande vise essentiellement à pouvoir surmonter ou contourner les obstacles à la mise en œuvre des interventions ou à concevoir des alternatives dans le cas où les obstacles sont reconnus comme insurmontables (éthiquement ou pratiquement). Par exemple, on demandera à l'anthropo-sociologie de fournir une méthode pour identifier a priori les effets pervers possibles d'une intervention donnée, de fournir une grille d'analyse permettant d'investiguer les raisons pour lesquelles des centres de santé ne sont pas utilisés, et de fournir les moyens pour augmenter l'utilisation de ces services, etc. Dans ce contexte, le recours à l'anthropologue ou au sociologue apparaît parfois comme le dernier avatar des solutions miracles à même de résoudre tous les problèmes de développement.

5 À côté de cette demande de type technique, il existe également une demande idéologique qui ne se reconnaît pas toujours comme telle. On attend ici du sociologue qu'illégitime et fasse apparaître comme scientifique les valeurs sous-jacentes à l'approche méthodologique élaborée par une équipe de recherche. Par exemple, on lui demandera de fonder scientifiquement la participation des acteurs à une méthode de planification.

6 Dans de tels contextes, une des premières tâches du sociologue/anthropologue au sein de l'équipe de recherche sera souvent d'expliciter et de reformuler la demande de manière à pouvoir la transformer en un authentique objet de recherche. Il lui faudra également, le cas échéant, réduire le niveau d'attente de ses collègues sous peine d'essuyer de profondes déconvenues lors de la poursuite de la recherche. Surtout, dans une perspective de collaboration à long terme, il lui faudra commencer un long processus d'explication de sa discipline elle-même, de ses fondements, de ses limites, etc. et, le cas échéant, entamer un travail sur les valeurs et les visions du monde de ses collègues techniciens. En effet, dans mon expérience, c'est surtout par ce biais que l'anthropo-sociologie peut se révéler la plus efficace. C'est en rendant explicite et en questionnant les valeurs qui sous-tendent certaines interventions que 
l'anthropo-sociologie peut amener des changements dans les manières de faire des techniciens ou des développeurs.

Objet des méthodologies

7 La recherche méthodologique appliquée au développement constitue un domaine vaste et varié et il ne peut être question ici d'en établir une liste exhaustive. Cependant, à titre d'illustration, signalons que dans le secteur de la santé, les méthodologies peuvent s'appliquer à des projets, des programmes, des systèmes, des politiques sectorielles ou intersectorielles. Elles peuvent concerner les différentes opérations du cycle des projets, programmes, etc., et s'appliquer à différents moments dans le temps. Les méthodes peuvent répondre à des besoins ponctuels ou continus dans le cadre d'une intervention (mise sur pied de systèmes d'information et de suivi, de procédures de négociation, etc.).

8 La recherche méthodologique peut viser également à mettre au point les outils et les procédures nécessaires pour répondre à des questions plus particulières telles que la durabilité, l'identification des effets pervers, la recherche d'indicateurs de "participation", l'analyse sociale, l'analyse culturelle, la formation et l'animation. Elle peut porter également sur les organisations engagées dans l'intervention et sur les procédures de transformation de ces organisations considérées de plus en plus comme faisant partie du problème du développement plutôt que de sa solution ${ }^{3}$.

Le champ de la recherche méthodologique est cependant en évolution constante. Cette évolution est due essentiellement aux changements qui apparaissent dans la théorie et la pratique du développement, et aux progrès réalisés dans la théorie des opérations (planification, évaluation, management, etc.) ${ }^{4}$. Les changements dans la conception théorique du développement et des projets conduisent à la création de demandes nouvelles. Par exemple, concevoir le développement comme un processus d'apprentissage ${ }^{5}$ entraîne de fait une demande de méthodologies appropriées. Il en va de même pour les opérations de projet, les projets participatifs requièrent des" évaluations participatives", etc. En évaluation, également, la recherche de la méthodologie idéale (et unique) qui prévalait dans les années 1970 (dessins quasi expérimentaux et analyses coûts-bénéfices) est progressivement abandonnée. Elle est de plus en plus remplacée par une diversification des approches et des techniques destinées à répondre aux multiples questions des différents acteurs du développement ${ }^{6}$.

Caractéristiques des méthodologies désirées

10 Les caractéristiques d'une méthode particulière sont liées au contexte pour lequel cette méthode est développée et à l'usage qui doit en être fait. Néanmoins, il semble que dans le secteur santé, un certain nombre de tendances actuelles en matière de recherche méthodologique peuvent être dégagées.

11 Une des premières préoccupations des opérateurs du développement est certainement l'opérationabilité de la méthode à élaborer. Il s'agit, en effet, de pouvoir disposer de méthodes non seulement efficaces mais praticables en pays en voie de développement c'est-à-dire pouvant être implantées sans personnel trop qualifié et sans matériel ou logistique excessivement sophistiqués, orientées vers la mise en œuvre des interventions, demandant peu de temps dans leur application et dans la production de résultats opérationnels, et enfin peu coûteuses en relation avec le coût total des opérations auxquelles elles s'appliquent. 

d'éléments de "participation", l'intersectorialité (la méthodologie doit permettre le dialogue entre différents secteurs ou disciplines), et la globalité (la prise en compte du contexte dans lequel se déroule l'intervention). La demande porte également sur des méthodes flexibles et adaptables à des situations locales spécifiques. Il s'agit ici de dégager un moyen terme entre d'une part, les approches standardisées applicables à de nombreuses situations (blueprints) et, d'autre part, le refus de toute méthodologie. Entre le questionnaire tout fait et le flou total, il s'agit de fournir les éléments et les outils aux agents de terrain pour qu'ils construisent eux-mêmes leurs propres grilles d'analyse, enquêtes, questionnaires, etc. ${ }^{7}$.

Caractéristiques de la recherche méthodologique ci-dessus, il est nécessaire de mener des recherches. La recherche méthodologique, lorsqu'elle est liée à l'opérationalisation de projets de développement, présente certaines caractéristiques, de fait ou souhaitées, que nous discutons ci-après.

De manière générale, la recherche méthodologique dans le domaine du développement n'est pas une recherche de pointe encore qu'elle peut être novatrice. Elle ne vise pas (ou ne devrait pas viser) à produire des techniques ou des approches sophistiquées et exclut les raffinements méthodologiques propres à la recherche scientifique classique. En effet, la recherche est toujours dépendante d'un contexte de développement donné ce qui implique de nombreux compromis entre exigences scientifiques et exigences opérationnelles. Les meilleures solutions techniques ne sont, en effet, généralement pas adaptées aux conditions du terrain. Par exemple, on ne pourra pas construire un indicateur de participation qui demande des mesures complexes sur le terrain: il faudra souvent se contenter d'un indicateur plus simple même si, dans l'absolu (scientifique), celui-ci est moins valide. D'autre part, les chercheurs peuvent difficilement imposer leurs exigences purement scientifiques aux organismes de financement.

15 En pratique, la recherche méthodologique procède essentiellement par accumulation d'expériences et ajustements successifs. Il en résulte que les projets de recherche sont généralement longs. Il ne faut pas seulement créer ou adapter des outils existants à des situations opérationnelles nouvelles, il faut également les tester dans des situations concrètes. Cela impliquera généralement plusieurs terrains d'expérimentation et donc de nombreux partenaires. Pour qui connaît la difficulté et le temps nécessaire pour établir de bonnes relations de travail entre différentes équipes de recherche, le fait que les financements portent le plus souvent sur le court terme est un obstacle important à ce type de recherche. Ces contraintes en font une recherche relativement coûteuse (en temps et en personnel). Pour les chercheurs, ceci entraîne la nécessité de pouvoir proposer aux organismes de financement un certain degré de validité externe de la méthode élaborée.

16 La recherche méthodologique est également une recherche précaire. Les caractéristiques jugées désirables évoluent, se modifient au cours du temps et peuvent rapidement démoder une méthode. Ces changements sont parfois justifiés. Toutefois, dans le monde du développement, les modes, les slogans, les acronymes et les dernières solutions miracles sont monnaie courante. Cela peut nuire à une certaine continuité dans la recherche. Il n'est pas rare non plus de voir "ré-étiqueter" des approches parfois anciennes avec des termes à la mode. 
17 En outre, la recherche méthodologique n'est pas toujours gratifiante en termes de prestige académique. Il existe peu de revues spécialisées francophones consacrées au sujet et beaucoup de résultats de recherche sont, dès lors, publiés dans la "littérature grise". Cela ne permet pas la critique par les pairs et ne garantit pas la valeur scientifique des travaux produits.

18 Finalement, la recherche méthodologique est de plus en plus le fait d'équipes interdisciplinaires suivant en cela la pratique des projets de développement Outre son adaptation à la réalité du développement, l'association de chercheurs de différentes disciplines permet d'éviter les dérives techniciennes ou académiques, et permet de mieux préciser l'objet de recherche. L'association des opérateurs du développement à la recherche, et ce, même lorsque les chercheurs ont une longue expérience de terrain, sert également de garde-fou. Les développeurs sont généralement plus "réalistes" et possèdent une vision plus exacte de ce qui est faisable et acceptable en matière de coût, de politique, etc.

Critères d'évaluation

19 Le contexte de développement dans lequel se déroule la recherche méthodologique requiert des critères d'évaluation de la recherche et de ses produits quelque peu différents de ceux de la recherche classique. En effet, au critère de qualité propre à toute recherche et pour lequel le jugement par les pairs reste sans doute le moyen le plus souhaitable de validation, viennent (ou devraient) s'ajouter d'autres critères importants.

20 Un critère qui me semble essentiel est l'utilisation effective du produit développé dans l'une ou l'autre intervention - ou tout au moins, et plus modestement, le déclenchement d'un intérêt chez les utilisateurs potentiels. En effet, à quoi bon produire des méthodologies si elles ne sont pas employées ? Ce critère permet également d'écarter du champ la pratique courante qui consiste à se servir de la recherche méthodologique comme prétexte pour mener une recherche de type plus fondamentale. Cette stratégie, si on peut la comprendre (les fonds destinés à la recherche en sciences humaines étant ce qu'ils sont..) est, à moyen terme, dommageable pour tous (chercheurs et opérateurs du développement). La solution à long terme passe par une (re)valorisation académique de la recherche (théorique et pratique) sur le développement Le système universitaire philippin pourrait constituer ici un modèle d'inspiration et de réflexion pour l'APAD. Il ajoute aux fonctions et aux rôles traditionnels de l'Université (formation et recherche) une fonction et une responsabilité de développement spécifique appelée "extension", qui permet aux chercheurs et au personnel enseignant, actifs sur le terrain, de développer des compétences et une reconnaissance à part entière dans l'institution.

21 D'autres critères d'évaluation importants de la recherche sont que la méthode élaborée soit plus efficace, ou que celle-ci présente des avantages comparatifs importants par rapport à d'autres méthodes employées précédemment. La contribution de la recherche au renforcement des capacités institutionnelles de recherche du pays en voie de développement dans lequel elle se déroule est également à prendre en considération. Ceci nécessite de travailler en partenariat avec des équipes de chercheurs locaux.

L'utilisation des produits de la recherche

22 Si l'on admet que le travail de recherche ne s'arrête pas (ou ne devrait en tout cas pas s'arrêter) à la publication des résultats de la recherche ou pire, à sa disparition dans un 
tiroir, se pose alors le problème de l'utilisation et/ou de la diffusion de la méthode élaborée par l'une ou l'autre agence de développement, ministère, ONG, etc. Il s'agit peut-être ici de l'étape la plus difficile dans le développement méthodologique. Autant dire que la qualité et les caractéristiques intrinsèques du "produit final" ne constituent pas, en eux-mêmes, un facteur de succès. Dans notre expérience, le fait que la recherche ait bénéficié d'un financement par une organisation internationale n'est pas non plus déterminant, encore que l'on puisse espérer des effets d'entraînement. Pour des raisons qui tiennent à certaines logiques bureaucratiques, les directions qui financent les recherches et les directions opérationnelles au sein d'une même organisation communiquent parfois peu entre elles - si elles ne sont pas en concurrence ouverte - avec cette conséquence paradoxale qu'une approche financée par une institution se voit finalement appliquée par une autre!

Les facteurs de succès à ce stade seront bien sûr l'expérience et la connaissance qu'ont les chercheurs de l'organisation, leur conviction et leur force de persuasion, certains talents en marketing et, souvent, la possibilité de lier la méthode à un programme de formation. Le plus important, cependant, sera l'implication et l'association de l'organisation à l'élaboration de toutes les étapes de la recherche. Il faudra pour cela emporter la conviction des spécialistes de l'organisation ainsi que de divers niveaux administratifs, qui ne sont pas nécessairement demandeurs de changement dans leurs procédures de travail.

C'est également à ce stade que la méthode élaborée rencontrera un risque d'écueil important: sa réduction, si ce n'est son détournement pur et simple, lors de son intégration dans les procédures administratives de l'organisation ${ }^{8}$. S'il n'existe pas de protection infaillible contre ce péril, certaines méthodes peuvent réduire le mal en incluant en elles-mêmes des éléments de sauvegarde.

En guise de conclusion...

25 La recherche méthodologique offre certainement un fort potentiel pour le développement d'une anthropologie du changement social et du développement Pour que cette recherche soit crédible cependant, les considérations opérationnelles devraient y tenir une place importante.

Il faut cependant reconnaître que l'anthropo-sociologie est techniquement limitée, et qu'elle est en soi incapable d'apporter des solutions à de nombreux problèmes. Mis à part la mise en œuvre des techniques de collecte de données propres aux sciences humaines, son rôle se limitera dès lors souvent à mettre en évidence les difficultés (ou à constater l'impossibilité) de l'intervention proposée, à identifier les divergences de valeur entre les différents acteurs du développement et, le cas échéant, à un travail sur certaines de ces valeurs.

Pour terminer sur une note positive, je voudrais mettre en avant deux caractéristiques de la recherche méthodologique qui me paraissent essentielles.

Premièrement, et contrairement à une idée parfois répandue, méthodologie n'est pas (toujours) synonyme de technocratie et/ou de bureaucratie. Les méthodologies peuvent être porteuses d'innovations et de changement dans la pratique du développement. Si la recherche méthodologique peut parfois être un refuge devant l'insuffisance des moyens ou être un prétexte à l'inaction (sous couvert d'accroître l'efficacité de l'action...), elle est peut-être plus à même de transformer concrètement les pratiques en matière de développement que les discours critiques et théoriques. La recherche méthodologique peut, en effet, offrir des approches alternatives et des outils pratiques 
et crédibles à des individus, ou à des groupes d'individus, porteurs de changement au sein des bureaucraties. Elle peut également entraîner des conséquences directes sur le terrain. Par exemple, l'introduction d'une méthode d'évaluation participative dans un projet (entendue ici comme l'association de tous les acteurs du projet au processus d'évaluation, y compris à la formulation des questions de l'évaluation et des recommandations) est susceptible de transformer radicalement tant le projet lui-même que l'esprit dans lequel celui-ci est mené. Et cela même dans des projets considérés comme participatifs...

Deuxièmement, le champ méthodologique est relativement dynamique et reste ouvert sur les débats plus théoriques qui traversent les sciences humaines. Si l'anthropologie et la sociologie théoriques peuvent et doivent y jouer un rôle, il serait cependant erroné de limiter la recherche méthodologique à une simple application de théories et de méthodes d'investigation classiques au terrain du développement. Non seulement parce que l'anthropologie-sociologie a jusqu'ici peu pris le développement comme objet, mais surtout parce que le champ méthodologique possède sa propre dynamique. Il connaît également ses ruptures paradigmatiques, ses écoles, etc. ${ }^{9}$ tout en ayant l'avantage de permettre de voir rapidement comment l'adoption d'une position paradigmatique donnée peut déboucher concrètement en termes de pratiques de développement.

\section{NOTES}

1.Lefèvre P., Beghin I. (Eds.) (1991). Guide to comprehensive evalualion of the nutritional aspects of projects and programmes. Health and community, Working paper $n^{\circ} 27$. Antwerp : Institute of Tropical Medicine. La recherche a été menée en collaboration avec le Collège d'Écologie Humaine de l'Université des Philippines à Los Banos et l'Institut Royal des Tropiques d'Amsterdam.

2.Tursz A., Cook J., De Bruycker M., Diesfeld H., Mercenier P. (Eds.), Methodology and Relevance of Health Systems Research., Paris : Centre International de l'Enfance (sous presse).

3.Par exemple : Foster G. M. (1987), "Bureaucratic aspects of international health agencies". Soc. Sci. Med, 25(9) : 1039-48.

4.Il est important de considérer que les divers champs de recherche méthodologique ont tendance à s'autonomiser. Ceci est fortement le cas aux États-Unis où l'évaluation, par exemple, est devenue une discipline autonome avec ses propres spécialistes, ses praticiens, ses associations professionnelles, son enseignement universitaire, ses revues scientifiques, etc.

5.Korten D. C. (1980) "Community organisation and rural development : a learning process approach", Public Administration Review, 40(5) : 480-512.

6.Binnendijk A. L. (1989), "Donor agency experiences with the monitoring and evaluation of development projects", Evaluation Review, 13(3) : 206-22.

7.Un bon exemple d'approche pas trop standardisée en nutrition est le guide de diagnostique rapide de Schrimshaw : malgré toutes les précautions prises par les 
auteurs qui insistent sur la nécessité d'adapter localement les outils proposés (grilles d'observation, questionnaires, etc.), ceux-ci ont toutes les chances d'être appliqués automatiquement à des situations non pertinentes. Schrimshaw S., Hurtado E. (1987), Rapid Assessment Procedures for Nutrition and Primary Health Care. Anthropological Approaches to Improving Programme Effectiveness. Tokyo : The United Nations University. 8.En Belgique, par exemple, beaucoup des critiques émises vis-à-vis de la méthode de Planification par Objectifs (PIPO) employée par l'Agence Générale de Coopération au Développement tiennent plus à son "mode d'administration" par l'AGCD qu'à ses caractéristiques propres. Le PIPO est dérivé de la méthodologie ZOPP (Ziel Orientierte Projekt Planung) développée par la coopération allemande (GTZ).

9.Voir par exemple dans le domaine de l'évaluation, le remarquable travail méthodologique de Guba qui dégage un paradigme d'évaluation constructiviste en opposition avec le paradigme positiviste : Guba E., Lincoln Y. (1989), Fourth Generation Evaluation, Beverly Hills, CA : Sage.

\section{AUTEUR}

PIERRE LEFÈVRE

Antwerpen, Belgique 